\title{
Intersubband plasmons in quasi-one-dimensional electron systems on a liquid helium surface
}

\author{
Marcos R. S. Tavares \\ Faculdade de Tecnologia da Baixada Santista, CEETPS, 11045-908 Santos, São Paulo, Brazil \\ G.-Q. Hai \\ Instituto de Física de São Carlos, Unversidade de São Paulo, 13560-970 São Carlos, São Paulo, Brazil \\ F. M. Peeters \\ Department of Physics, University of Antwerp (UIA), B-2610 Antwerpen, Belgium \\ Nelson Studart \\ Departamento de Física, Universidade Federal de São Carlos, 13565-905 São Carlos, São Paulo, Brazil \\ (Received 6 June 2003; published 15 October 2003)
}

\begin{abstract}
The collective excitation spectra are studied for a multisubband quasi-one-dimensional electron gas on the surface of liquid helium. Different intersubband plasmon modes are identified by calculating the spectral weight function of the electron gas within a 12 subband model. Strong intersubband coupling and depolarization shifts are found. When the plasmon energy is close to the energy differences between two subbands, Landau damping in this finite-temperature system leads to plasmon gaps at small wave vectors.
\end{abstract}

PACS number(s): 73.21.-b, 73.43.Lp

The electron system on the surface of liquid helium discovered about 30 years ago has provided an ideal platform to study many-body effects in low dimensions. ${ }^{1,2}$ Recently, quasi-one-dimensional (Q1D) electron gas embedded in such structures has been realized in laboratory by bending the liquid helium surface. ${ }^{3}$ In some sense, this Q1D system is similar to those in semiconductor quantum wires, nanotubes, and metallic chains where many-body effects have been extensively studied. ${ }^{4}$ However, this new system on the surface of liquid helium provides us more degrees of freedom to explore many-body effects in a 1D framework. It is free from impurities, being much clearer than other systems. Furthermore, it is of a wider variable range of electron densities. Several theoretical and experimental studies have been carried out on the electron transport and electron-electron interactions in this system. ${ }^{5,6}$ The electron system on the liquid helium surface is achieved at finite temperatures and the electron density is usually much smaller than that in semiconductor structures. In most cases, it is considered as a nondegenerate electron gas obeying the Maxwell-Boltzmann statistics. ${ }^{7}$ On the other hand, the $1 \mathrm{D}$ confinement is weak and the gap between the 1D subbands is of the same order as the thermal energy. As consequence, many subbands are usually occupied forming a real multisubband Q1D electron system. Very recently, dispersion relation of the collective excitations (plasmons) was studied theoretically within the random-phase approximation (RPA) for the Q1D electron gases on the surface of liquid helium. ${ }^{7,8}$ The RPA is believed to be a more reliable approximation to study collective excitations of such a nondegenerate Q1D electron gas in the classical regime. In these studies, ${ }^{8}$ a two-subband model was used and particular attention was devoted to the dispersion relations of one intrasubband and one intersubband plasmon modes found within this model. Notice that, both the RPA and the quasicrystalline approximation, being valid in opposite limits of electron density at fixed temperature, result in very similar plasmon dispersions indicating that the RPA could be correct over a wide range of electron densities.

This Rapid Communication is focused on the collective excitation spectra of the multisubband Q1D electron gas on the surface of liquid helium. Special attention is paid to the intersubband coupling and effects of the single-particle excitations (SPE's), i.e., the Landau damping, on the plasmon modes at finite temperature. We study these elementary excitations by calculating the full spectral weight function of this classical electron system. The Landau damping induced phenomena are expected to be of a different kind, provided the electrons are in a very different regime from those embedded in semiconductor quantum wires. In a Q1D electron system, the intersubband interactions are much stronger than those in higher dimensions. Furthermore, temperature effects are important because the energy gap between the 1D electron subbands is comparable to the thermal energy $k_{B} T$. Many subbands can be occupied even for small densities and temperatures. As a consequence, the intersubband coupling should be treated properly. In the calculations, we take into account as many subbands as possible to guarantee the efficiency of the method and to make sure that the obtained plasmon spectra are independent of the number of subbands included.

We consider the same structure for the Q1D electron system as in Ref. 7 where the electron mobility was studied. The 1D confinement on the liquid helium is determined by both the surface curvature radius in the $y$ direction $R=5$ $\times 10^{-4} \mathrm{~cm}$ and the so-called holding field applied in the $z$ direction $E_{\perp}=3 \times 10^{3} \mathrm{~V} / \mathrm{cm}$. This confinement is then approximated by a parabolic potential in the $y$ direction with a confinement frequency $\omega_{0}=\sqrt{e E_{\perp} / m R}$, where $m$ and $e$ are the mass and the charge of the electron, respectively. The energy eigenvalues of an electron in the system are given by $E_{n}\left(k_{x}\right)=k_{x}^{2} / 2 m+\left(n-\frac{1}{2}\right) \omega_{0}$, where $k_{x}$ is the electron wave vector in the $x$ direction and $\left(n-\frac{1}{2}\right) \omega_{0}$ are the electron energy levels due to confinement in the $y$ direction, with $n$ 
$=1,2, \ldots$, being the subband index. Here, we consider the electron gas being of zero thickness in the $z$ direction because the energy gap between the two lowest levels due to confinement in this direction is greater than $30 \mathrm{~K},{ }^{9}$ whereas the confinement energy $\omega_{0}$ in the $y$ direction is less than $1 \mathrm{~K}$. Another important parameter is the localization length $y_{0}$ $=k_{0}^{-1}=\sqrt{1 / 2 m \omega_{0}}$ of the electrons in the $y$ direction (we consider $\hbar=1$ throughout this paper).

Our calculations show that there is only one observable intrasubband plasmon mode but many intersubband modes in the present Q1D electron system. All the observable intersubband plasmon modes are related to the first and second subbands denoted by $(1, n)$ and $(2, n)$, respectively, where $n=1,2,3, \ldots$. Such intersubband modes are important since most of the electrons occupy states in the two lowest subbands and intersubband interactions are strong in a onedimensional geometry. Furthermore, we find that intersubband coupling between the higher subbands and the two lowest subbands affects significantly the low-energy intersubband plasmon modes. A correct theoretical consideration on such a coupling turns out to be essential in obtaining even the lowest intersubband plasmon modes. In order to consider this effect properly, we use in this work a 12 subband model so that the plasmon spectra shown below do not change anymore when more subbands are included. Moreover, we show that pronounced Landau damping occurs when the plasmon energy approaches the energy difference $n \omega_{0}$ between two subbands leading to gaps in the plasmon spectra.

A traditional way to obtain the plasmon dispersion relations in Q1D electron gases at zero temperature ${ }^{10}$ is to find the roots of the equation $\operatorname{det}\left|\operatorname{Re}\left\{\varepsilon_{\alpha \beta}(q, \omega)\right\}\right|=0$, where $\varepsilon_{\alpha \beta}$ is the multisubband dielectric matrix and the indices $\alpha$ $\equiv\left(i, i^{\prime}\right)$ and $\beta \equiv\left(j, j^{\prime}\right)$ with the subband indices $i, i^{\prime}, j$, and $j^{\prime}$. However, this method cannot provide us complete information on the plasmon excitations in the present system. There are two main reasons for that: (i) it is difficult to figure out where the plasmon excitations are Landau damped by SPE's because $\operatorname{Im}\left\{\varepsilon_{\alpha \beta}(q, \omega)\right\} \neq 0$ in the whole $\omega-q$ plane at finite temperatures; and (ii) the thermal fluctuations in the system might easily populate several subbands even for small densities. Therefore, intersubband interactions can be strong so that a reasonable amount of subbands should be included in the calculation. The equation $\operatorname{det}\left|\operatorname{Re}\left\{\varepsilon_{\alpha \beta}\right\}\right|=0$ yields many roots but does not provide information on the relative importance of each one. Thus, one cannot distinguish which roots correspond to the plasmon modes.

A more reliable way to study the collective excitations in such a multisubband system at finite temperatures is to calculate the so-called spectral weight ${ }^{11}$

$$
S(q, \omega)=-\sum_{\alpha \beta} \operatorname{Im}\left[\varepsilon_{\alpha \beta}^{-1}(q, \omega) \Pi_{\beta}^{\delta}(q, \omega)\right] .
$$

The peaks of this function give information on the plasmon excitations and, from their position, we can obtain the dispersion relations of the plasmon modes. By showing all excitation modes through $S(q, \omega)$, one provides a very efficient guide of what should be observable in the experiments. These observations are certainly dependent on external probes (e.g., light polarization). Equation (1) also involves the 1D noninteracting irreducible polarizability function $\Pi_{\beta}^{\delta}(q, \omega)$. We remember that the Maxwell-Boltzmann distribution function is used in calculating $\Pi_{\beta}^{\delta}(q, \omega)$, with $\delta$ being a phenomenological constant which is responsible for broadening of the energy levels $E_{n}\left(k_{x}\right)$ mainly due to ripplon and evaporated helium atom scattering on the surface. The quantity $S(q, \omega)$ is directly related to the optical (such as inelastic light scattering spectra) and transport (such as conductivity) properties. The multisubband dielectric matrix function

$$
\varepsilon_{\alpha \beta}(q, \omega)=\delta_{\alpha \beta}-V_{\alpha \beta}(q) \Pi_{\beta}^{\delta}(q, \omega),
$$

is written within the RPA, with $V_{\alpha \beta}(q)$ being the Coulomb electron-electron bare interaction.

The symmetric confinement potential in the $y$ direction leads to the electron-electron Coulomb interaction $V_{\alpha \beta}(q)$ $=0$ when $i+i^{\prime}+j+j^{\prime}$ is an odd number. Consequently, the dielectric matrix elements (both the real and the imaginary parts) $\varepsilon_{\alpha \beta}(q, \omega)=0$ for $i+i^{\prime}+j+j^{\prime}=$ odd. The dielectric matrix can then be decoupled into two submatrices $\varepsilon_{\alpha \beta}^{\text {even }}(q, \omega)$ and $\varepsilon_{\alpha \beta}^{\text {odd }}(q, \omega)$ with both $i+i^{\prime}$ and $j+j^{\prime}$ being even and odd numbers, respectively. The even (odd) dielectric submatrix involves only intersubband electron-electron interaction for one electron from subband $i$ to subband $i^{\prime}$ with $i+i^{\prime}=$ even $\left(i+i^{\prime}=\right.$ odd $)$ and the other from subband $j$ to $j$ with $j+j^{\prime}=$ even $\left(j+j^{\prime}=\right.$ odd $)$. As a consequence, the spectral weight function can be treated separately in two parts $S(q, \omega)=S^{\text {even }}(q, \omega)+S^{\text {odd }}(q, \omega)$.

In order to understand the damping induced phenomena in the present system, we calculate first the spectral weight of the single-particle excitations $S_{s p}(q, \omega)=$ $-\Sigma_{j j^{\prime}} \operatorname{Im}\left[\Pi_{j j^{\prime}}^{\delta}(q, \omega)\right]$. It is straightforward to analytically obtain the imaginary part of the polarizability $\Pi_{\beta}^{\delta}(q, \omega)$ at finite temperatures. ${ }^{8}$ Considering the level broadening effects, it is given by

$$
\begin{aligned}
\operatorname{Im}\left[\Pi_{i j}^{\delta}\right]= & N_{e}\left\{\exp [-j / T] I^{\delta}\left(\xi_{i j}^{(+)}\right)-\exp [-i / T] I^{\delta}\left(\xi_{i j}^{(-)}\right)\right\} / \\
& q \sqrt{\pi T}[1+\operatorname{coth}(1 / 2 T)],
\end{aligned}
$$

where $N_{e}$ is the total electron density, $T$ the temperature, $q$ the wave vector in the $x$ direction, and

$$
I^{\delta}(\xi)=\Delta \int_{-\infty}^{\infty} d k_{x} \frac{\exp \left[-\left(k_{x}+\xi\right)^{2}\right]}{k_{x}^{2}+\Delta^{2}}
$$

with $\xi_{i j}^{( \pm)}=\left[\omega+(i-j) \pm q^{2}\right] / 2 q \sqrt{T}$ and $\Delta=\delta / 2 q \sqrt{T}$. The wave vectors $k_{x}$ and $q$ are written in units of $k_{0}$, while the frequency $\omega$, the broadening constant $\delta$ and the temperature $T$ are in units of $\omega_{0}$. Notice that, in the limit $\delta \rightarrow 0$, the integral $I^{\delta}(\xi)=\pi \exp \left(-\xi^{2}\right)$, which is a Gaussian function of $\xi$. We take the broadening parameter $\delta=10^{-2} \omega_{0}$ throughout this paper. This value corresponds to realistic electron mobilities due to ripplon scattering. ${ }^{7,12}$

In Fig. 1 we show $S_{s p}(q, \omega)$ as a function of $\omega$ for different values of the wave vector $q$. Here, the temperature $T$ $=0.4 \mathrm{~K}$, the electron density $N_{e}=10^{4} \mathrm{~cm}^{-1}$ and the holding field $E_{\perp}=3 \times 10^{3} \mathrm{~V} / \mathrm{cm}$. The energy gap between the quan- 


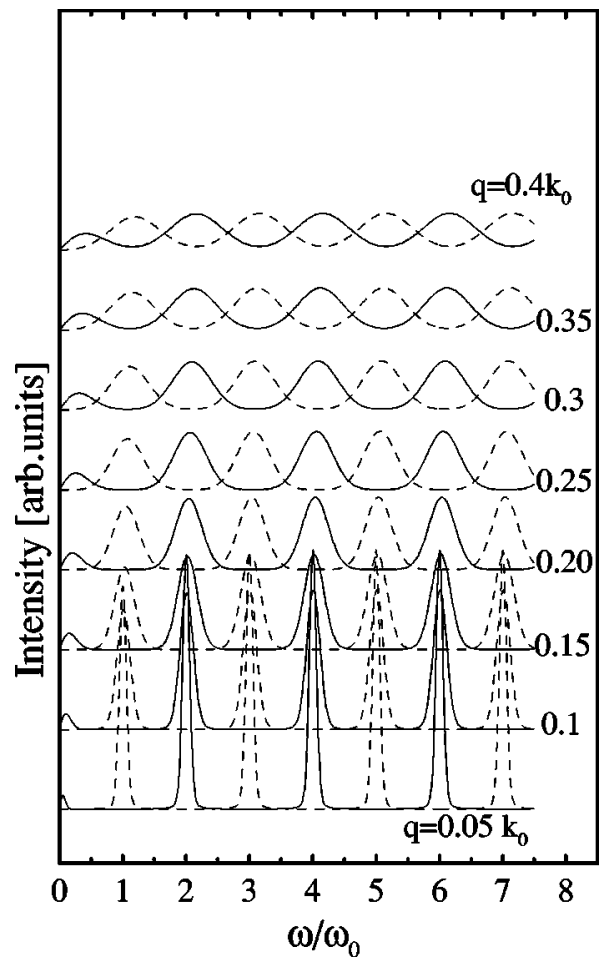

FIG. 1. Spectral weights of the single-particle excitations, $S_{s p}^{\text {even }}(q, \omega)$ (solid curves) and $S_{s p}^{\text {odd }}(q, \omega)$ (dashed curves), for $T$ $=0.4 \mathrm{~K}$ and $N_{e}=10^{4} \mathrm{~cm}^{-1}$ with different wave vectors q as indicated.

tized levels at this holding field is $\omega_{0} \simeq 0.784 \mathrm{~K}$ which leads to electron occupation up to the second and the third subbands of about $14 \%$ and $2 \%$, respectively, in relation to the first one. For the sake of consistency, we plot $S_{s p}(q, \omega)$ in two parts: $S_{s p}^{\text {even }}(q, \omega)=-\Sigma_{j+j^{\prime}=\operatorname{even}} \operatorname{Im}\left[\Pi_{j j^{\prime}}^{\delta}(q, \omega)\right] \quad$ (solid curves) and $S_{s p}^{\text {odd }}(q, \omega)=-\Sigma_{j+j^{\prime}=\text { odd }} \operatorname{Im}\left[\Pi_{j j^{\prime}}^{\delta}(q, \omega)\right]$ (dashed curves). For small wave vector $q$, the spectral weights of the intersubband SPEs are mostly described by Gaussian functions centered at energies $\omega=n \omega_{0}$, where $n=1,2,3, \ldots$ On the other hand, the intrasubband SPE's are represented by the less weighted peak seen in the lower energy part of the spectra. This peak is mostly due to excitations in the first subband. Figure 1 show us that SPE induced effects should be significant at $\omega=n \omega_{0}$ for small $q$.

We show in Fig. 2 the spectral weights (a) $S^{\text {even }}(q, \omega)$ and (b) $S^{\text {odd }}(q, \omega)$ for different electron densities $N_{e}$ at a small fixed wave vector $q=0.1 k_{0}$. The temperature is taken as $T=0.4 \mathrm{~K}$. The density varies from $N_{e}=1 \times 10^{3} \mathrm{~cm}^{-1}$ (lowest curve) until $N_{e}=1.45 \times 10^{4} \mathrm{~cm}^{-1}$ (top curve) with a difference $1.5 \times 10^{3} \mathrm{~cm}^{-1}$. For $N_{e}=1 \times 10^{3} \mathrm{~cm}^{-1}$, only the first subband has a significant electron density so the plasmon modes related to this subband can be observed. This help us to identify the different peaks corresponding to the intrasubband mode $(1,1)$ and intersubband modes $(1,3)$, $(1,5)$, and $(1,7)$ in Fig. 2(a). The peaks in Fig. 2(b), at the same electron density, are due to the plasmon modes $(1,2)$, $(1,4)$, and $(1,6)$. As the density increases, these peaks shift to higher energy because the depolarization shift is enhanced. Meanwhile, the intersubband modes related to the second

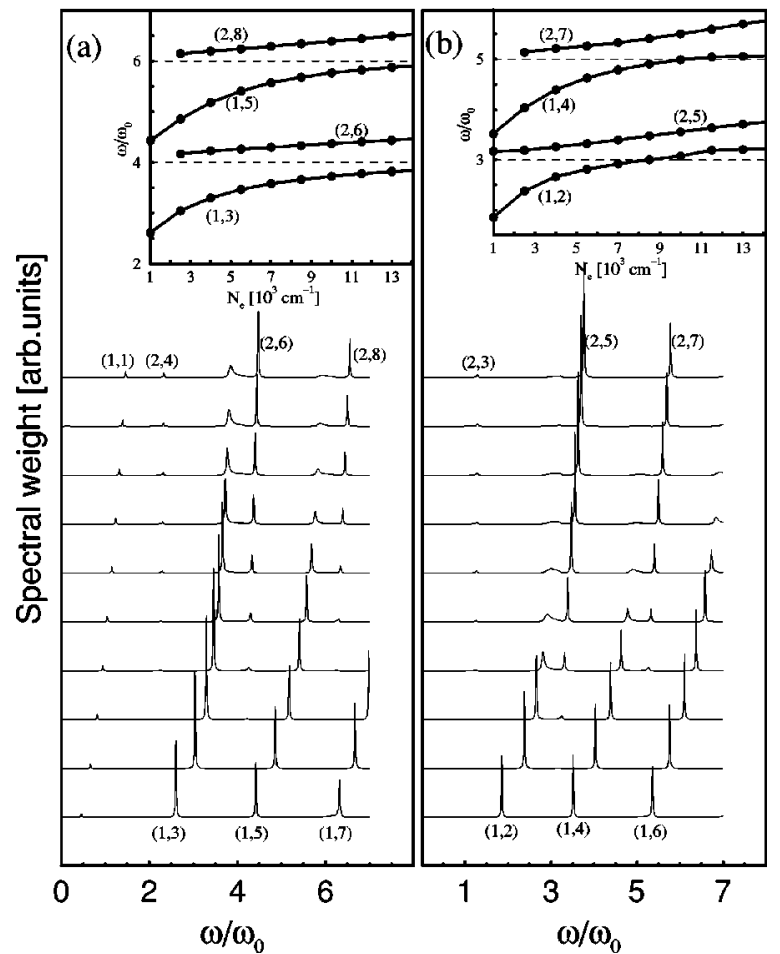

FIG. 2. The spectral weights (a) $S^{\text {even }}(q, \omega)$ and (b) $S^{\text {odd }}(q, \omega)$ for $q=0.1 k_{0}$ and $T=0.4 \mathrm{~K}$. The electron densities are from $N_{e}$ $=1 \times 10^{3} \mathrm{~cm}^{-1}$ (lowest curve) to $N_{e}=1.45 \times 10^{4} \mathrm{~cm}^{-1}$ (top curve) with a difference $1.5 \times 10^{3} \mathrm{~cm}^{-1}$. The insets indicate the position of the peaks as a function of $N_{e}$.

subband appear as indicated in the figure. We also observe that the Landau damping becomes stronger when the plasmon mode $(1, n)$ approaches the frequency $(n+1) \omega_{0}$ where the single-particle excitations are of high intensity as shown in Fig. 1. The insets show the energy position of the peaks due to the intersubband plasmons as a function of the density $N_{e}$. The gaps result from the Landau damping and are clearly seen around the energies $\omega=4 \omega_{0}$ and $6 \omega_{0}$ in Fig. 2(a); and $\omega=3 \omega_{0}$ and $5 \omega_{0}$ in Fig. 2(b).

In Fig. 3, we plot the spectral weight function for different $q$ values corresponding to those in Fig. 1 with $N_{e}$ $=10^{4} \mathrm{~cm}^{-1}$ and $T=0.4 \mathrm{~K}$. Figures 3(a) and 3(b) show $S^{\text {even }}(q, \omega)$ and $S^{\text {odd }}(q, \omega)$, respectively. The dispersion relations of the plasmon modes obtained from the peak position are given in the insets. We also see small peaks in the full spectra due to single-particle excitations indicated by the open dots in the insets. The lowest branch (open dots) represents the intrasubband SPEs. Similarly as in Q1D Fermi liquid electron systems at zero temperature, with increasing $q$, the spectral weight of the intrasubband (intersubband) plasmon mode increases (decreases). We also observe the Landau damping induced gaps appearing around $\omega=n \omega_{0}$ at small $q$ where the SPE's are strong. Furthermore, the peaks due to the plasmon excitations become wider and lower at larger $q$ because $S_{s p}(q, \omega)$ tends to be a more uniform function of $\omega$.

For the sake of completeness, we analyze in Fig. 4 the spectral weight $S(q, \omega)$ as a function of the energy $\omega$ and for several temperatures. Here, the density $N_{e}=10^{4} \mathrm{~cm}^{-1}$ and 


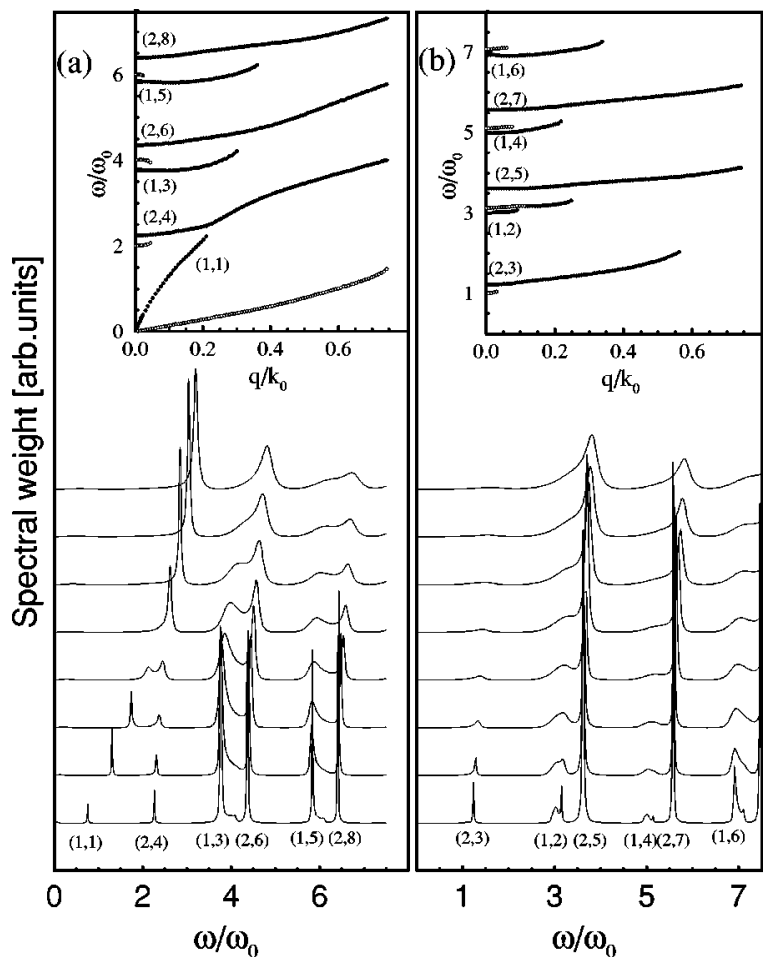

FIG. 3. The spectral weights (a) $S^{\text {even }}(q, \omega)$ and (b) $S^{\text {odd }}(q, \omega)$ for $T=0.4 \mathrm{~K}, N_{e}=10^{4} \mathrm{~cm}^{-1}$, and different wave vectors corresponding to those in Fig. 1. The insets give the dispersion relation of the plasmon modes. The open dots indicate the small peaks due to single-particle excitations.

$q=0.1 k_{0}$. From the bottom to the top curves, the temperature increases from $T=0.2 \mathrm{~K}$ to $2.0 \mathrm{~K}$ with a step of $0.2 \mathrm{~K}$. As the temperature increases, the peaks of the intersubband plasmon modes $(1, n)$ related to the first subband shift to lower frequency while those modes $(2, n)$ related to the second subband shift to higher frequency. Such a change is mainly induced by the redistribution of the electron density in different subbands. As temperature increases, the electron density of the first subband decreases but that of the higher subband increases. The decrease of the electron density in the first subband results in a decrease of the depolarization shift of the related intersubband plasmon modes $(1, n)$. The insets show the energy position of these peaks as a function of temperature.
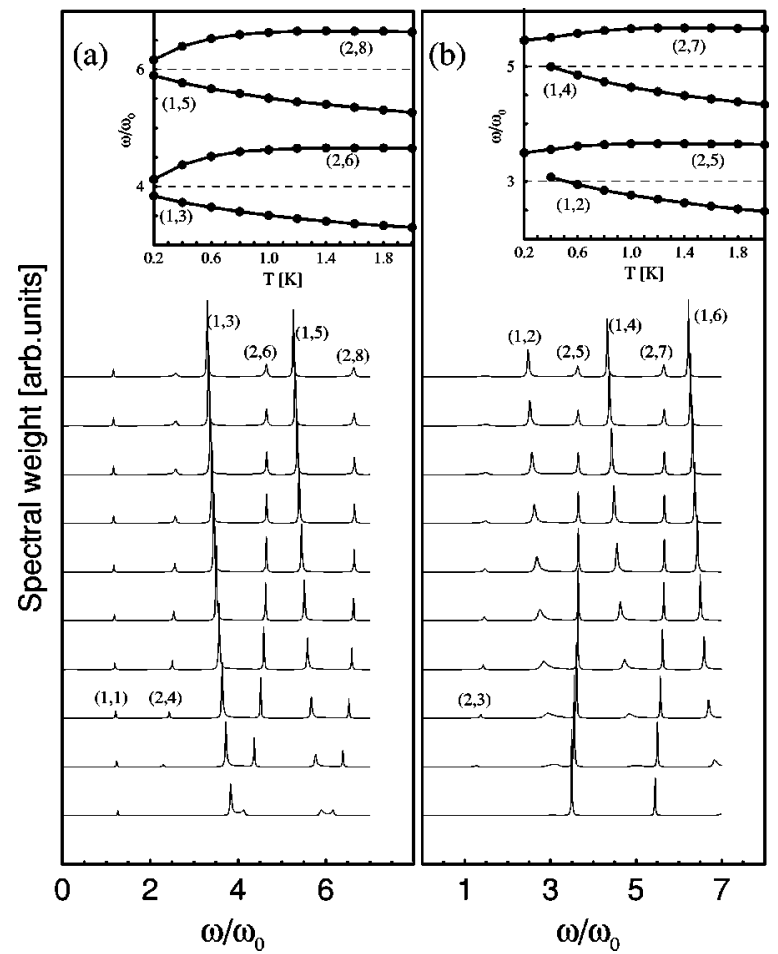

FIG. 4. The spectral weights (a) $S^{\text {even }}(q, \omega)$ and (b) $S^{\text {odd }}(q, \omega)$ for $q=0.1 k_{0}$ and $N_{e}=10^{4} \mathrm{~cm}^{-1}$. The temperature increases from $T=0.2 \mathrm{~K}$ (the lowest curve) to $2.0 \mathrm{~K}$ (the top curve) with a step of $0.2 \mathrm{~K}$. The insets show the position of the peaks.

In summary, we investigated the plasmon modes of the Q1D electron systems on the surface of liquid helium. We used a multisubband approach and treated the system as a classical nondegenerate gas obeying Maxwell-Boltzmann statistics. We found strong intersubband plasmon modes related to the first two subbands. The single-particle excitations in the system are responsible for strong Landau damping at frequencies $\omega=n \omega_{0}$ where gaps appear in the plasmon spectra.

This work was supported by FAPESP (the research foundation agency of the state of São Paulo), CNPq (Brazil), IUAP-V, GOA (Antwerp, Belgium) and the European Community's Human Potential Program under Contract No. HPRN-CT-2000-00157 "Surface electrons."
${ }^{1}$ M.W. Cole, Rev. Mod. Phys. 46, 451 (1974); Two-Dimensional Electron Systems in Helium and Other Substrates, edited by E. Y. Andrei (Kluwer, Dordrecht, 1997).

${ }^{2}$ C.C. Grimes and G. Adams, Phys. Rev. Lett. 36, 145 (1976).

${ }^{3}$ Yu.Z. Kovdrya and V.A. Nikolaenko, Fiz. Nizk. Temp. 18, 1278 (1992).

${ }^{4}$ J. Voit, Rep. Prog. Phys. 58, 977 (1994).

${ }^{5}$ Yu.Z. Kovdrya, Low Temp. Phys. 29, 77 (2003).

${ }^{6}$ P. Glasson, V. Dotsenko, P. Fozooni, M.J. Lea, W. Bailey, G. Papageorgiou, S.E. Andresen, and A. Kristensen, Phys. Rev. Lett. 87, 176802 (2001).

${ }^{7}$ S.S. Sokolov, G.Q. Hai, and N. Studart, Phys. Rev. B 51, 5977
(1995).

${ }^{8}$ S.S. Sokolov and N. Studart, Phys. Rev. B 57, R704 (1998); J. Phys.: Condens. Matter 12, 9563 (2000); S.S. Sokolov and O.I. Kirichek, Low Temp. Phys. 20, 599 (1994).

${ }^{9}$ D.K. Lambert and P.L. Richards, Phys. Rev. B 23, 3282 (1981).

${ }^{10}$ Marcos R.S. Tavares and G.-Q. Hai, J. Phys.: Condens. Matter 13, 6421 (2001); G.-Q. Hai and Marcos R.S. Tavares, Phys. Rev. B 61, 1704 (2000).

${ }^{11}$ For a review, see G. D. Mahan, Many Particle Physics (Plenum, New York, 1981).

${ }^{12}$ Yu.Z. Kovdrya, V.A. Nikolaenko, and S.P. Gladchenko, Physica B 284, 168 (2000) 